\title{
Ultraviolet photoluminescence from ferromagnetic Fe-doped AIN nanorods
}

\author{
X. H. Ji, S. P. Lau, ${ }^{\text {a) }}$ S. F. Yu, H. Y. Yang, and T. S. Herng \\ School of Electrical and Electronic Engineering, Nanyang Technological University, Nanyang Avenue, \\ Singapore 639798, Singapore \\ A. Sedhain, J. Y. Lin, and H. X. Jiang \\ Department of Physics, Kansas State University, Manhattan, Kansas 66506-2601 \\ K. S. Teng \\ Multidisciplinary Nanotechnology Centre, School of Engineering, University of Wales Swansea, Singleton \\ Park, Swansea SA2 8PP, United Kingdom \\ J. S. Chen \\ Data Storage Institute, 5 Engineering Drive 1, Singapore 117608, Singapore and Department of Material \\ Science and Engineering, National University of Singapore, Singapore 119260, Singapore
}

(Received 13 February 2007; accepted 18 April 2007; published online 9 May 2007)

\begin{abstract}
Fe-doped AlN (AlN:Fe) nanorods on silicon substrates were fabricated using a catalysis-free vapor phase method. The AlN:Fe nanorods exhibited high crystalline quality and preferred $c$-axis orientation. The spontaneous saturated magnetization of the AlN:Fe nanorods was determined to be $\sim 0.64 \mu_{B} / \mathrm{Fe}$ at room temperature. Room temperature photoluminescence measurement of the AlN:Fe nanorods revealed two strong ultraviolet emissions at 3.69 and $6.02 \mathrm{eV}$ which could be attributed to $\mathrm{Fe}^{3+}$-related and band edge emissions, respectively. The Fe-doped AlN nanorods not only exhibited ferromagnetism but also significantly enhanced the band edge emission as compared to the undoped AIN nanorods. (C) 2007 American Institute of Physics. [DOI: 10.1063/1.2738370]
\end{abstract}

Diluted magnetic semiconductors (DMSs) are attractive materials for the spintronics, as they can have the inherent properties of both semiconductor and ferromagnetic materials. The most direct method to induce spin-polarized electrons into a semiconductor is by introducing appropriate transition metals (TMs) or rare-earth dopants to produce a DMS. Meanwhile, the integration of future spintronic devices into nanodevices would require the fabrication of onedimensional (1D) DMS nanostructures in well-defined architectures. ${ }^{1}$ The behavior of carriers in nanostructures may also allow for the enhancement of the ferromagnetic semiconductor behavior, because nanostructures are expected to have longer coherence times than in the bulk, which may provide a pathway for increasing the spin lifetime in ferromagnetic semiconductor devices for practical applications. ${ }^{2}$ Besides the well studied oxides and III-V DMSs, group III nitrides are getting more attractive because of the theoretical prediction ${ }^{3}$ and experimental realization of room temperature ferromagnetism. Although AlN-based DMS bulk materials have been extensively studied and modeled, ${ }^{4-8}$ there are very few reports on the synthesis of 1D nanostructures ${ }^{9}$ because of the limited TM equilibrium solubility in semiconductors as well as in the intrinsic difficulty in nanocrystal doping. So far, TM dopants in AIN such as $\mathrm{Mn}, \mathrm{Cr}, \mathrm{Co}$, and $\mathrm{Cu}$ have been employed. Meanwhile, $\mathrm{Fe}^{3+}$ $\left(3 d^{5}\right)$ having a high magnetic moment of $5 \mu_{B} /$ Fe could lead to the high spin state. ${ }^{10}$ In this work, magnetic and photoluminescence (PL) properties of Fe-doped AlN nanorods were investigated.

AlN:Fe nanorods were prepared in a horizontal tube furnace. The experimental procedure has been reported elsewhere. ' Briefly, aluminum chloride powder $\left(\mathrm{AlCl}_{3}\right.$, Sigma-Aldrich, 99.99\%), ferric chloride anhydrous $\left(\mathrm{FeCl}_{3}\right.$,

${ }^{\text {a)} E l e c t r o n i c ~ m a i l: ~ e s p l a u @ n t u . e d u . s g ~}$
Sigma-Aldrich), and ammonium $\left(\mathrm{NH}_{3}\right)$ were used as sources. AlN:Fe nanorods were grown on Si substrate without catalyst at $1000{ }^{\circ} \mathrm{C}$ for about $2 \mathrm{~h}$. Crystallography of the nanorods was determined by using Siemens D5005 x-ray diffractometer (XRD) with $\mathrm{Cu} K \alpha$ irradiation at $40 \mathrm{kV}$ and $40 \mathrm{~mA}$. Field emission scanning electron microscopy (SEM, JEOL JSM-6340F) and transmission electron microscopy (TEM, JEOL JEM-2010) were used for the morphologies and structural analysis. X-ray photoelectron spectroscopy (XPS, VG ESCAlab MKII) was engaged using Al $K \alpha$ as $\mathrm{X}$-ray source to analyze the element content. Deep ultraviolet PL spectroscopy under a $197 \mathrm{~nm}$ laser excitation was employed to study the optical properties of the nanorods at room temperature. Magnetic hysteresis loops of the AlN:Fe nanorods were measured by an alternating gradient magnetometer with a maximum field of $10 \mathrm{kOe}$.

Representative XRD pattern of the AlN:Fe nanorods is shown in Fig. 1(a). Several diffraction peaks were observed, which corresponds to the wurtzite AlN structure. No diffraction peaks of $\mathrm{Fe}-\mathrm{N}$ or $\mathrm{Al}-\mathrm{Fe}$ compounds were found within the sensitivity of XRD. The strong (002) intensity indicates that the AlN:Fe nanorods preferentially grow along the $c$ axis. The inset of Fig. 1 is the typical cross-sectional SEM image of the AlN:Fe nanorods. It is observed that a high density AlN:Fe nanorod array was grown either perpendicular or slantingly from the wafer to form the quasiarrays. These nanorods are $\sim 500 \mathrm{~nm}$ in length with decreasing diameters along the growth direction. Figure 1(b) shows the TEM image of the AlN:Fe nanorods which exhibits a sharp tip of $15-20 \mathrm{~nm}$ in diameter. The corresponding highresolution TEM images and the selected area electron diffraction as shown in Fig. 1(c) reveal that the nanorod is a single crystal with a spacing of $0.49 \mathrm{~nm}$ between the neighboring lattice planes [the distance between the neighboring 

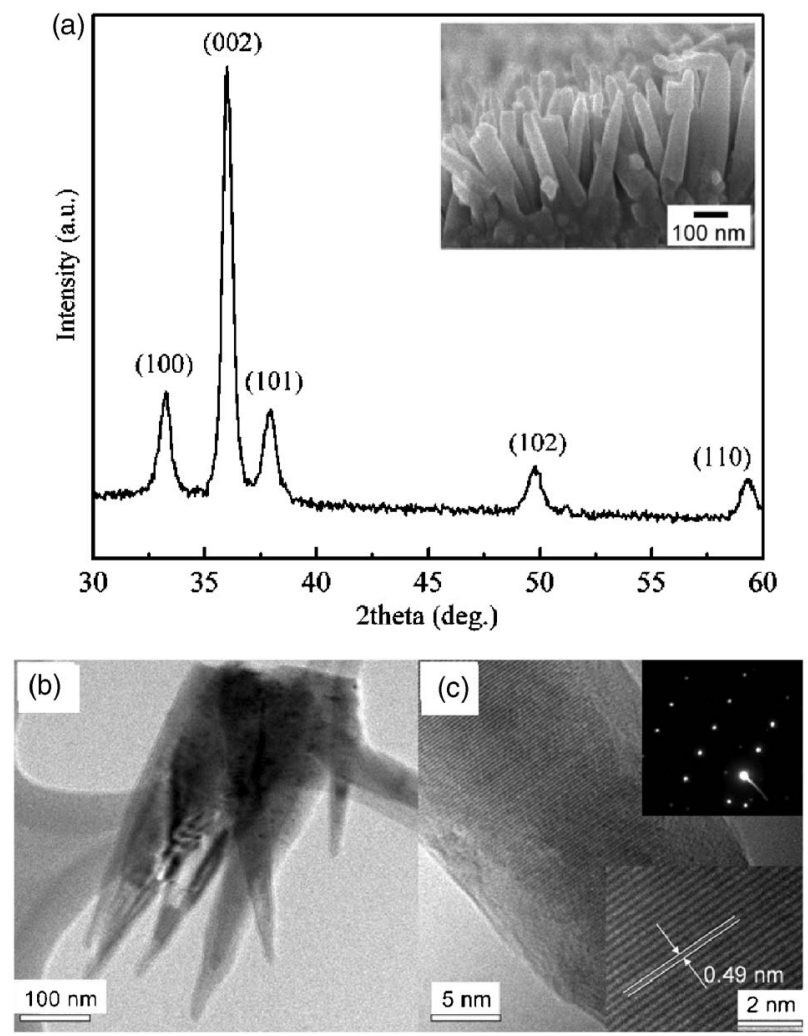

FIG. 1. (a) XRD spectrum of the AIN:Fe nanorods. The inset is the crosssectional SEM image of AlN:Fe nanorods. (b) TEM image of the AlN:Fe bundle. (c) A single nanorod with the corresponding electron diffraction pattern and high-resolution image.

lattice planes is noted as $d_{(h k l)}$, which is in agreement with $d_{(001)}$ of hexagonal AlN.

Figure 2 shows the XPS spectra of the undoped and Fe-doped AlN nanorods. Besides the absorbed carbon and oxygen, $\mathrm{Al}$ and $\mathrm{N}$ were clearly evident from both spectra. The atomic ratios of N/Al of undoped and Fe-doped AlN are estimated to be 0.91 and 0.79 , respectively, suggesting that the AlN nanorods are $\mathrm{Al}$ rich. The atomic concentration of $\mathrm{Fe}$ is about $3.5 \%$ in the Fe-doped AlN nanorods. It is noted that the binding energy of $\mathrm{Fe} 2 p_{3 / 2}$ is located at $710.19 \mathrm{eV}$, which indicates that $\mathrm{Fe}$ ions are in high spin state. ${ }^{11}$

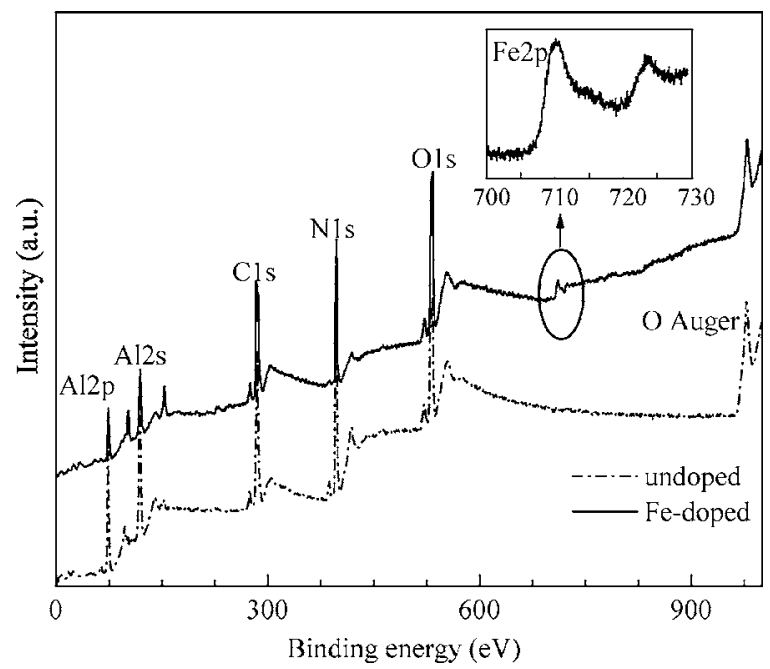

FIG. 2. XPS spectra of the undoped and Fe-doped AlN nanorods. The inset shows the magnification of $\mathrm{Fe} 2 p$ peak. hows the magnification of $\mathrm{Fe} 2 p$ peak.
Downloaded 12 Jul 2010 to 129.118.86.45. Redistribution subject to AlP license or copyright; see http://apl.aip.org/apl/copyright.jsp

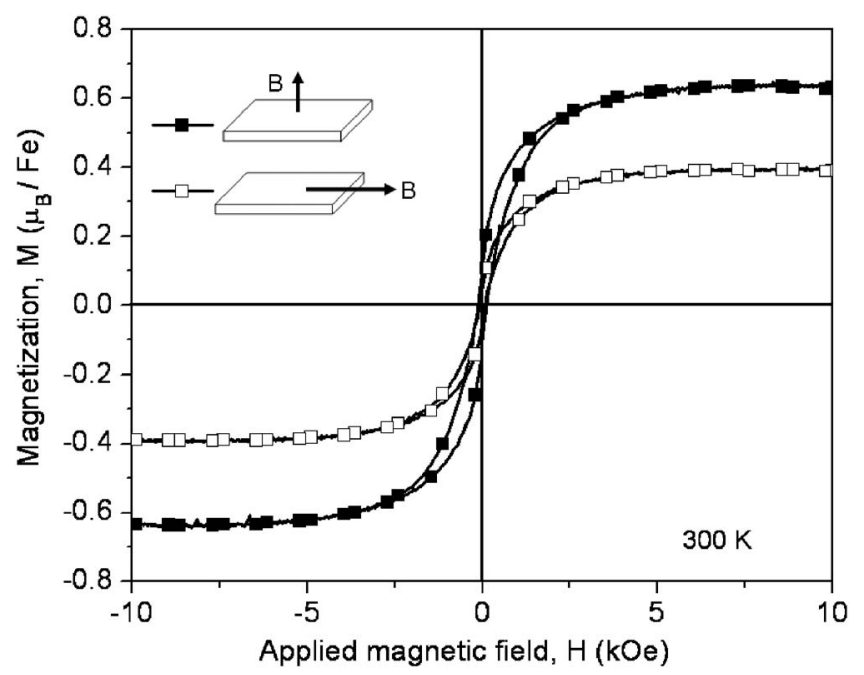

FIG. 3. Magnetization hysteresis loops of the AlN:Fe nanorods measured at room temperature with applied field parallel and perpendicular to the sample.

The magnetization versus magnetic field $(M-H)$ curve of the AlN:Fe nanorods measured at room temperature shows a hysteresis loop, revealing its ferromagnetic behavior, as shown in Fig. 3. The spontaneous saturated magnetization and coercivity of the AlN:Fe are estimated to be $\sim 0.64 \mu_{B} / \mathrm{Fe}$ and 116 Oe when the field is applied perpendicular to the sample, which is $\sim 61 \%$ and $50 \%$ larger than the field applied parallel to the sample, respectively. The magnetic anisotropy in TM substituted semiconductor is a common characteristic of DMSs. ${ }^{12}$ This indicates that a significant fraction of Fe spins are coupled magnetically in AlN, and the saturated magnetic moment of the AlN:Fe nanorods is significantly larger than Fe-implanted $\mathrm{ZnO}$ (Ref. 13) and Cr-doped AlN. ${ }^{14}$

The $\mathrm{Fe}^{3+}-V_{\mathrm{N}^{-}} \mathrm{Fe}^{3+}$ groups are expected in the structure. An electron trapped in the nitrogen vacancy $\left(V_{\mathrm{N}}\right)$ constitutes an $F$ center, where the electron occupies an orbital which overlaps with the $d$ shell of both ion neighbors. Since $\mathrm{Fe}^{3+}$, $3 d^{5}$, only has unoccupied minority spin orbitals, the trapped electron will be spin down $(\downarrow)$ and the two ion neighbors spin up $(\uparrow)$, resulting in the magnetization. Although no Ferelated secondary phases can be detected by the XRD and TEM, we cannot rule out the possibility of nanosized Fe clusters or Fe oxide phases which may also contribute to the ferromagnetism.

PL emission from the AlN and AlN:Fe nanorods is shown in Fig. 4. The PL spectrum of the undoped AlN nanorods comprises two strong impurity-related emission lines at 3.25 and $4.32 \mathrm{eV}$, and a weak emission at $5.83 \mathrm{eV}$. The emission lines of 3.25 and $4.32 \mathrm{eV}$ can be attributed to the transitions between free or donor bound electrons to $\mathrm{Al}$ vacancy and vacancy-impurity complex $\left(V_{\mathrm{Al}}\right.$ complex $)$ with different charge states. ${ }^{15}$ However, the emission line of $5.83 \mathrm{eV}$, which is much smaller than the room temperature band edge emission (for example, $5.96 \mathrm{eV}$ ) (Ref. 16) of AlN, is likely to be related to an unidentified impurity as the peak position does not shift with temperatures from 300 down to $10 \mathrm{~K}$. Further experiments are currently underway to determine the origin of this peak. It is noted that both impurity emissions are absent from the $\mathrm{Fe}$-doped nanorods, but a $3.96 \mathrm{eV}$ emission becomes apparent in addition to the band edge emission at $6.02 \mathrm{eV}$. The vanishing of the 3.25 and 


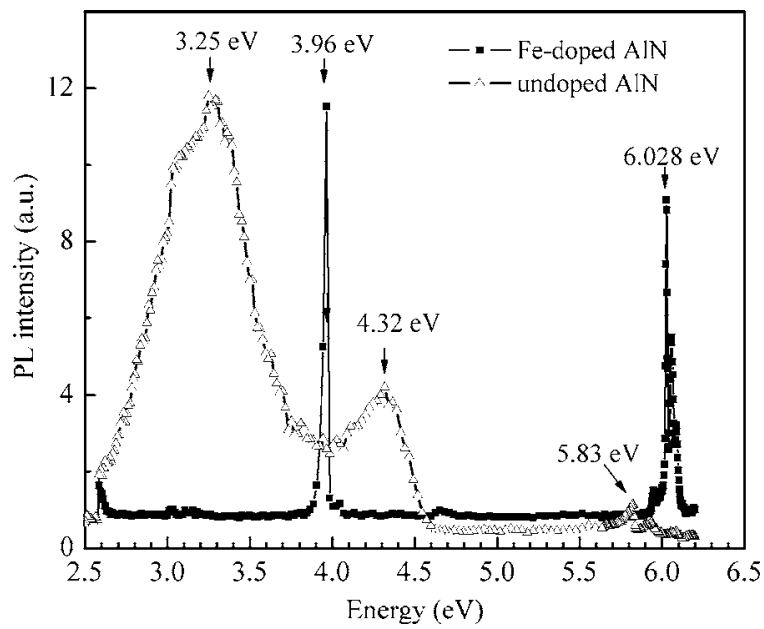

FIG. 4. PL spectra of the undoped and Fe-doped AIN nanorods.

$4.32 \mathrm{eV}$ emissions may be due to the formation of the nonradiative defect complex induced by Fe ions. Heitz et al. ${ }^{17}$ reported that the deep $\mathrm{Fe}^{3+/ 2+}$ acceptor level is $3.17 \mathrm{eV}$ above the valence band maximum in GaN. Assuming band offsets in nitrides following the internal reference rule, the $\mathrm{Fe}^{3+/ 2+}$ acceptor level is expected to be about $3.96 \mathrm{eV}$ above the valence band of AlN, where the valence band offset for GaN/AlN is $0.79 \mathrm{eV} .^{18}$ Therefore, the emission line of $3.96 \mathrm{eV}$ with narrow linewidth $(18.5 \mathrm{meV})$ might be related to $\mathrm{Fe}^{3+/ 2+}$ acceptor. The narrow UV emission linewidth of the Fe-doped AIN is very similar to that observed in Gdimplanted AlN epilayer. ${ }^{19}$ Therefore, strong dopant-related UV emissions not only can take place in AIN thin films but also in nanostructures. Besides the observed ferromagnetism, Fe doping also seems to play an important role in enhancing the UV emissions in AIN nanorods. AlN:Fe may be a promising DMS material for spintronics.

In summary, Fe-doped AlN nanorods exhibited room temperature ferromagnetism with a saturated magnetic moment of $0.64 \mu_{B} / \mathrm{Fe}$ when the field was applied perpendicular to the sample. The two UV emission peaks at 3.96 and $6.02 \mathrm{eV}$ from the Fe-doped AIN nanorods could be attributed to $\mathrm{Fe}^{3+/ 2+}$ acceptor and band edge emission, respectively. It is noted that Fe doping plays a significant role in enhancing the UV emissions in the AlN nanostructures.
This work was partially supported by NTU RGM 17/04 and MOE ARC 2/06. The research at KSU was supported by a grant from DOE. One of the authors (H.Y.Y.) acknowledges the support from Singapore Millennium Foundation Fellowships.

${ }^{1}$ Y. Xia, P. Yang, Y. Sun, Y. Wu, B. Mayers, B. Gates, Y. Yin, F. Kim, and H. Yan, Adv. Mater. (Weinheim, Ger.) 15, 353 (2003).

${ }^{2}$ M. H. Kane, M. Strassburg, A. Asghar, Q. Song, S. Gupta, J. Senawiratne, C. Hums, U. Haboeck, A. Hoffmann, D. Azamat, W. Gehlhoff, N. Dietz, Z. J. Zhang, C. J. Summers, and I. T. Ferguson, Proc. SPIE 5732, 389 (2005).

${ }^{3}$ T. Dietl, H. Ohno, F. Matsukura, J. Cibert, and D. Ferrand, Science 287, 1019 (2000).

${ }^{4}$ D. Kumar, J. Antifakos, M. G. Blamire, and Z. H. Barber, Appl. Phys. Lett. 84, 5004 (2004).

${ }^{5}$ Y. Endo, T. Sato, A. Takita, Y. Kawamura, and M. Yamamoto, IEEE Trans. Magn. 41, 2718 (2005).

${ }^{6}$ H. X. Liu, S. Y. Wu, R. K. Singh, L. Gu, D. J. Smith, N. Newman, N. R. Dilley, L. Montes, and M. B. Simmonds, Appl. Phys. Lett. 85, 4076 (2004).

${ }^{7}$ H. Katayama-Yoshida and K. Sato, J. Phys. Chem. Solids 64, 1447 (2003).

${ }^{8}$ S. Y. Wu, H. X. Liu, L. Gu, R. K. Singh, L. Budd, M. Schilfgaarde, W. R. McCartney, D. J. Smith, and N. Newman, Appl. Phys. Lett. 82, 3047 (2003).

${ }^{9}$ X. H. Ji, S. P. Lau, S. F. Yu, H. Y. Yang, T. S. Herng, and J. S. Chen, Nanotechnology 18, 105601 (2007).

${ }^{10}$ X. Y. Cui, B. Delley, A. J. Freeman, and C. Stampfl, Phys. Rev. Lett. 97, 016402 (2006).

${ }^{11}$ L. N. Mazalov, I. P. Asanov, and V. A. Varnek, J. Electron Spectrosc. Relat. Phenom. 96, 209 (1998).

${ }^{12}$ M. Venkatesan, C. B. Fitzgerald, J. G. Lunney, and J. M. D. Coey, Phys. Rev. Lett. 93, 177206 (2004).

${ }^{13}$ K. Potzger, Shengqiang Zhou, H. Reuther, A. Mücklich, F. Eichhorn, N. Schell, W. Skorupa, M. Helm, J. Fassbender, T. Herrmannsdörfer, and T. P. Papageorgiou, Appl. Phys. Lett. 88, 052508 (2006).

${ }^{14}$ J. Zhang, X. Z. Li, B. Xu, and D. J. Sellmyer, Appl. Phys. Lett. 86, 212504 (2005).

${ }^{15}$ T. Marttila and R. M. Nieminen, Phys. Rev. B 55, 9571 (1997).

${ }^{16}$ J. Li, K. B. Nam, M. L. Nakarmi, J. Y. Lin, and H. X. Jiang, Appl. Phys. Lett. 81, 3365 (2002).

${ }^{17}$ R. Heitz, P. Maxim, L. Eckey, P. Thurian, A. Hoffmann, I. Broser, K. Pressel, and B. K. Meyer, Phys. Rev. B 55, 4382 (1997).

${ }^{18}$ N. Nepal, M. L. Nakarmi, H. U. Ang, J. Y. Lin, and H. X. Jiang, Appl. Phys. Lett. 89, 192111 (2006).

${ }^{19}$ J. M. Zavada, N. Nepal, J. Y. Liu, H. X. Jiang, E. Brown, U. Hömmerich, J. Hite, G. T. Thaler, C. R. Abernathy, S. J. Pearton, and R. Gwilliam, Appl. Phys. Lett. 89, 152107 (2006). 